54. The varieties of self-projective quartic and quintic curves have been tabulated for the general case by Ciani and Snyder respectively. Dr. Winger in his paper presents the projectively distinct types of the most general rational curves of these orders which are invariant under the different finite collineation groups. The quartics are readily obtained from the consideration of the Stahl binary sextic. Six types are found with characteristic groups of orders $2,3,4,4,6$ and 24 , the first three being cyclic, besides one with an infinite group.

Of the quintics, two admit a one-parameter group. The others belong to cyclic groups of orders $2,3,4,5$ (two types), and dihedral groups of orders 4, 6, and 10 (two types),--eleven in all.

F. N. Cole, Secretary.

\title{
THE PRODUCT OF TWO OR MORE GROUPS.
}

BY PROFESSOR G. A. MILLER.

(Read before the American Mathematical Society, December 31, 1912.)

\section{§1. Introduction.}

If $H_{1}$ and $H_{2}$ are any two groups, the symbol $H_{1} \cdot H_{2}$ denotes the totality of the products obtained by multiplying each operator of $H_{1}$ on the right by every operator of $H_{2}$. A necessary and sufficient condition that this totality constitutes a group is that $H_{1} \cdot H_{2} \equiv H_{2} \cdot H_{1}$. As $H_{1} \cdot H_{2}$ is always composed of the inverses of all the operators represented by $H_{2} \cdot H_{1}$, irrespective of whether this product is a group or does not have this property, we may also say that a necessary and sufficient condition that $H_{1} \cdot H_{2}$ is a group is that it includes the inverse of each one of its operators.

Suppose that $H_{1}$ and $H_{2}$ have exactly $h_{0}$ operators in common. These common operators constitute a subgroup $H_{0}$, which is known as the cross-cut of $H_{1}$ and $H_{2}$. It is easy to prove that the number of the distinct operators in $H_{1} \cdot H_{2}$ is always $h_{1} h_{2} / h_{0}$, where $h_{1}$ and $h_{2}$ represent the orders of $H_{1}$ and $H_{2}$ respectively. To see that $H_{1} \cdot H_{2}$ cannot involve more than this number of distinct operators, it is only neces- 
sary to arrange all the operators of $H_{1}$ and $H_{2}$ in augmented left and right co-sets, ${ }^{*}$ respectively, às follows:

$$
\begin{aligned}
& H_{1}=H_{0}+s_{2} H_{0}+s_{3} H_{0}+\cdots+s_{n_{1}} H_{0}, \\
& H_{2}=H_{0}+H_{0} t_{2}+H_{0} t_{3}+\cdots+H_{0} t_{n_{2}} .
\end{aligned}
$$

The fact that $h_{1} h_{2} / h_{0}$ of the operators in $H_{1} \cdot H_{2}$ are distinct results from the following equations:

If

then

$$
s_{a} H_{0} \cdot H_{0} t_{\beta}=s_{a_{1}} H_{0} \cdot H_{0} t_{\beta_{1}}
$$

$$
s_{a_{1}}{ }^{-1} s_{a} H_{0}=H_{0} t_{\beta_{1}} t_{\beta}{ }^{-1} \text {. }
$$

As the first member of the last equation represents an operator of $H_{1}$ while the second member represents an operator of $H_{2}$, it results that the last equation implies $\alpha_{1}=\alpha$ and $\beta_{1}=\beta$. Hence the elementary theorem: If $H_{1}$ and $H_{2}$ have exactly $h_{0}$ common operators, then $H_{1} \cdot H_{2}$ involves $h_{1} h_{2} / h_{0}$ distinct operators, and each of these operators appears exactly $h_{0}$ times among the operators of $\mathrm{H}_{1} \cdot \mathrm{H}_{2}$.

While the theory of the product of two groups is very elementary, the theory of the product of more than two groups is much more complex. We observe in the first place that if $H_{1}, H_{2}, \cdots, H_{\lambda}$ represent any $\lambda$ groups, then $H_{1} \cdot H_{2} \cdots H_{\lambda}$ and $H_{\lambda} \cdots H_{2} \cdot H_{1}$ are composed of operators which are respectively the inverses of each other, independently of whether these products are groups or do not have this property. If one of these products is a group, the other is evidently also a group. Moreover, it is clear that $H_{1} \cdot H_{2} \cdots H_{\lambda}$ is a group whenever its factors $H_{1}, H_{2}, \cdots, H_{\lambda}$ can be permuted according to all of the substitutions of the cyclic group generated by the substitution $\left(H_{1} H_{2} \cdots H_{\lambda}\right)$, without affecting the value of this product. In fact, if the function $H_{1} \cdot H_{2} \cdots H_{\lambda}$ admits all the substitutions of this cyclic group, the product of any two of its operators is again an operator in this product since

$$
\begin{aligned}
H_{1} \cdot H_{2} \cdots & H_{\lambda} \cdot H_{1} \cdot H_{2} \cdots H_{\lambda}=H_{1} \cdot H_{2} \cdots H_{\lambda} \cdot H_{1} \cdot H_{2} \cdots H_{\lambda-1} \\
& =H_{1} \cdot H_{2} \cdots H_{\lambda-1} \cdot H_{\lambda} \cdot H_{1} \cdots H_{\lambda-2}=\cdots=H_{1} \cdot H_{2} \cdots H_{\lambda} .
\end{aligned}
$$

Hence the theorem: If the product $H_{1} \cdot H_{2} \cdots H_{\lambda}$ admits the cyclic group on its factors, in order, it must also admit the dihedral group on these factors.

* Transactions Amer. Math. Society, vol. 12 (1911), p. 326. 


\section{§2. Substitutions which Transform a Product of Groups into Itself.}

One of the most useful theorems in the theory of substitution groups is often stated in a somewhat indefinite form as follows: All the substitutions on $n$ letters which transform into itself a given function of these letters constitute a group. Hence it may be desirable to direct attention to an important limitation of this general statement of the theorem, which exhibits interesting properties of the product of three groups. The main point in question can be illustrated by means of the simple group of order 60, which we shall represent by the symbol $G$ throughout the present section.

Let three Sylow subgroups of $G$ whose orders are 3,4 , and 5 be represented by the symbols $G_{1}, G_{2}$, and $G_{3}$ respectively, and suppose that $G_{1}$ and $G_{2}$ have been so selected that they generate a subgroup of order 12 while $G_{3}$ is any one of the subgroups of order 5 contained in $G$. From the fact that the number of the distinct operators in the product of two groups which have only identity in common is equal to the product of the orders of these groups, it results that every operator of $G$ is found once and only once in each of the products $G_{1} \cdot G_{2} \cdot G_{3}, G_{3} \cdot G_{1} \cdot G_{2}$.

The second of these products may be obtained from the first by means of the substitution $\left(G_{1} G_{3} G_{2}\right)$, and hence we may say that $G=G_{1} \cdot G_{2} \cdot G_{3}$ is transformed into itself by this substitution. We proceed to prove that $G$ is not transformed into itself by the square of this substitution, and hence it will result that the substitutions on the letters $G_{1}$, $G_{2}, G_{3}$ which transform $G$ into itself do not constitute a group. This fact will be established if we prove that $G \neq G_{2} G_{3} G_{1}$.

We proceed to prove the more general theorem that $G$ cannot be the product of three Sylow subgroups of different orders if the middle one of these subgroups is of order 5 . Since the 60 operators of $G_{1} \cdot G_{3} \cdot G_{2}$ are the inverses of those of $G_{2} \cdot G_{3} \cdot G_{1}$, it is only necessary to prove that it is impossible to find in $G$ three Sylow subgroups $G_{2}, G_{3}, G_{1}$ of orders 4, 5, 3 respectively such that $G=G_{2} \cdot G_{3} \cdot G_{1}$.

If $G$ were equal to $G_{2} \cdot G_{3} \cdot G_{1}$, all the transforms of this product under the symmetric group of order 120 would also be equal to $G$. Since all the subgroups of order 4 in $G$ are conjugate, we may select any one of these five subgroups for $G_{2}$. If we represent $G$ as the alternating group of degree 5 , it 
may therefore be supposed that

$$
G_{2}=1,(a b)(c d),(a c)(b d),(a d)(b c) .
$$

The substitutions which transform both $G$ and $G_{2}$ into themselves transform also the six subgroups of order 5 contained in $G$ among themselves, and hence we may assume that

$$
G_{3}=1,(a b c d e),(a c e b d),(a d b e c),(a e d c b) .
$$

The four substitutions 1, $(a d)(b c)$, $(a b d c)$, (acdb), which transform each of the three groups $G, G_{2}, G_{3}$ into itself, transform also the ten subgroups of order 3 contained in $G$ into three complete sets of conjugates, two sets being composed of four subgroups each, while the remaining set involves only two such subgroups. Hence it remains only to prove that $G$ cannot be represented by any one of the following three products of three Sylow subgroups of different orders:

$$
\begin{aligned}
& \begin{array}{l|l|ll|l|l}
1 & 1 & 1 & 1 & 1 & 1 \\
(a b)(c d) & (a b c d e) & (a b c) & (a b)(c d) & (a b c d e) & (a b e) \\
(a c)(b d) & (a c e b d) & (a c b) & (a c)(b d) & (a c e b d) & (a e b) \\
(a d)(b c) & (a d b e c) & & (a d)(b c) & (a d b e c) & \\
(a e d c b) & & & (a e d c b) &
\end{array} \\
& \begin{array}{l|l|l}
1 & 1 & 1 \\
(a b)(c d) & (a b c d e) & (a d e) \\
(a c)(b d) & (a c e b d) & (a e d) . \\
(a d)(b c) & (a d b e c) & \\
(a e d c b) &
\end{array}
\end{aligned}
$$

The fact that none of these products represents 60 distinct operators results immediately from the following equations:

$$
\begin{aligned}
& (a c)(b d)(a b c d e)(a c b)=(a d b e c),(a c)(b d)(a e b)=(a c e b d), \\
& (a c)(b d)(a b c d e)(a d e)=(a e d c b) .
\end{aligned}
$$

Hence it results that the substitutions on $G_{1}, G_{2}, G_{3}$ which transform the product $G_{1} \cdot G_{2} \cdot G_{3}$ into itself do not constitute a group.* The theorem stated at the beginning of this section, relating to all the substitutions which transform a given func-

* This theorem is closely related to the theorem that all the substitutions which transform a function into those having the same numerical value do not always constitute a group. 
tion into itself, applies therefore only to a special class of functions. In particular, it applies to the formal values of rational functions of the roots of an equation. In fact, it was first formulated with a view to these functions.

\section{§3. Groups which are Products of Sylow Subgroups.}

The illustrative example above directs attention to groups which are the product of non-conjugate Sylow subgroups.* It is evident that every group whose order is of the form $p^{a} q^{\beta}, p$ and $q$ being prime numbers, is the product of any two arbitrary Sylow subgroups of orders $p^{\alpha}$ and $q^{\beta}$ respectively. On the other hand, the icosahedral group is the product of Sylow subgroups provided these subgroups occur in a given order and have been properly chosen. The question whether a group is a product of Sylow subgroups or does not have this property is, in general, very complex when the number of the distinct prime factors of the order of the group exceeds two. Even in the case when the number of these factors is only three there are great difficulties. We proceed to give a few theorems relating to this case.

Suppose that the order of $G$ is $p^{a} q^{\beta} r^{\gamma}$, where $p, q, r$ are three distinct prime numbers, and let $G_{1}, G_{2}, G_{3}$ be three Sylow subgroups of orders $p^{a}, q^{\beta}, r^{\gamma}$ respectively. From the facts that the two double co-sets $G_{1} s_{1} G_{3}$ and $G_{1} s_{2} G_{3}$, where $s_{1}$ and $s_{2}$ are any operators of $G$, either have no operator in common or have all their operators in common, and that the number of the distinct operators in each of these double co-sets is a multiple of each of the numbers $p^{a}$ and $r^{\gamma}$, it results that the number of the distinct operators in $G_{1} \cdot G_{2} \cdot G_{3}$ is always of the form $p^{\alpha} q^{\beta} r^{\gamma}-k p^{a} r^{\gamma}$.

A necessary and sufficient condition that $G=G_{1} \cdot G_{2} \cdot G_{3}$ is that $k=0$ in the formula which closes the preceding paragraph, and a necessary and sufficient condition that this $k=0$ is that the equation $G_{1} s G_{3}=s$ has exactly $q^{\beta}$ solutions when $s$ represents successively each of the operators of $G_{2}$ once and only once. In other words, a necessary and sufficient condition that $k=0$ is that $G_{1} s G_{3}=s$, where $s$ represents any operator of $G_{2}$, can be solved only when the operators from $G_{1}$ and $G_{3}$ are both identity. If $G_{1} s G_{3}$ has exactly $n$ operators

\footnotetext{
* Some properties of these groups were determined by E. Maillet, Bull.
} Soc. Math. de France, vol. 28 (1900), p. 7. 
in common with $G_{2}, s$ being some operator of $G_{2}$, then each of these $n$ operators occurs exactly $n$ times in $G_{1} \cdot G_{2} \cdot G_{3}$. In fact, each of the $p^{\alpha} r^{\gamma}$ distinct operators of $G_{1} s G_{3}$ occurs exactly $n$ times in $G_{1} \cdot G_{2} \cdot G_{3}$. Hence

$$
k=\frac{n_{1}-1}{n_{1}} m_{1}+\frac{n_{2}-1}{n_{2}} m_{2}+\cdots+\frac{n_{\lambda}-1}{n_{\lambda}} m_{\lambda},
$$

where $m_{a}(\alpha=1,2, \cdots, \lambda)$ is the exact number of the distinct operators of $G_{2}$ which occur $n_{a}$ times in $G_{1} \cdot G_{2} \cdot G_{3}$. It is clear that $m_{\alpha}$ is always a multiple of $n_{\alpha}$.

If $G$ is any solvable group, it is known that we reach identity by forming the successive commutator subgroups, and that the group $H$ which precedes identity in this series of commutator subgroups is an invariant abelian sub-group of $G$. If we can prove that $G$ is a product of non-conjugate Sylow subgroups, provided we assume that the quotient group $G / H$ has this property, we can evidently establish by complete induction that every solvable group is a product of Sylow subgroups.

Suppose that $G / H$ is the product of non-conjugate Sylow subgroups. To every Sylow subgroup of order $p^{a}$ in $G / H$ there corresponds at least one Sylow subgroup of order $p^{\beta}$ in $G$. If we select any set of Sylow subgroups of $G$ which correspond, in order, to the set of such subgroups whose product is $G / H$, we evidently obtain a set of non-conjugate Sylow subgroups of $G$ whose product, in order, constitutes all the operators of $G$. Hence we have established the interesting theorem: Every solvable group is the product of non-conjugate Sylow subgroups, and the order of the factors in this product is arbitrary.

While every solvable group is the product of non-conjugate Sylow subgroups, it is not true that a group which is a product of non-conjugate Sylow subgroups is always solvable, as may be seen from the case of the icosahedral group cited above. A more interesting example is furnished by the simple group of order 360, whose non-conjugate Sylow sub-groups are of orders 5, 8, 9 respectively. Although this group does not contain a subgroup whose order is the product of two of the orders of its Sylow subgroups, yet it is possible to find three non-conjugate Sylow subgroups such that their product gives this group. In fact, it is not difficult to verify that the fol- 
lowing product gives this simple group in the form of the alternating group on six letters:

\begin{tabular}{l|l|l}
1 & 1 & 1 \\
$(b c e d f)$ & $(a b)(c d)$ & $(a c f)$ \\
$(b e f c d)$ & $(a c)(b d)$ & $(a f c)$ \\
$(b d c f e)$ & $(a d)(b c)$ & $(b d e)$ \\
$(b f d e c)$ & $(a c)(e f)$ & $(a c f)(b d e)$ \\
& $(b d)(e f)$ & $(a f c)(b d e)$ \\
& $(a b c d)(e f)$ & $(b e d)$ \\
& $(a d c b)(e f)$ & $(a c f)(b e d)$ \\
& & $(a f c)(b e d)$.
\end{tabular}

It is not possible to select three non-conjugate subgroups $G_{1}, G_{2}, G_{3}$ such that the simple group $G$ of order 360 is their product, if the middle factor is either of order 5 or of order 9 . That is, if $G=G_{1} \cdot G_{2} \cdot G_{3}$, it is necessary that the order of $G_{2}$ is 8. Hence the product $G_{1} \cdot G_{2} \cdot G_{3}=G$ is transformed into itself only by the substitution $\left(G_{1} G_{3}\right)$ and identity. The substitutions which transform this product into itself must therefore constitute a group. The proof of the fact, stated above, that $G$ cannot be the product of three nonconjugate Sylow subgroups if the order of the middle factor is either 5 or 9 , is not difficult when $G$ is represented as the alternating group on six letters, but it is somewhat long and hence we omit it.

The preceding results give rise to two important questions which remain unanswered. The first of these may be stated as follows: Is there a simple group of composite order which is the product of each one of its possible sets of non-conjugate Sylow subgroups? If this question can be answered negatively, then it follows from what precedes that a necessary and sufficient condition that a group is solvable is that it is the product of each one of its possible sets of non-conjugate Sylow subgroups, taken in every possible order. It is evident that the simple group of order 168 is the product of some sets of non-conjugate Sylow subgroups taken in any one of the six possible orders, but it is not the product of every possible set of non-conjugate Sylow subgroups, since it contains two operators of orders 2 and 3 respectively whose product is of order 7, as was observed by Dyck.* A group which is the product of each one of its possible sets of non-conjugate Sylow

*Dyck, Math. Annalen, vol. 20 (1882), p. 41. 
subgroups cannot involve two operators whose orders are powers of prime numbers and whose product has an order which is a power of another prime number. In particular, a solvable group cannot involve two such operators.

The second question to which we referred above is as follows: Is there a group which is not the product of some one of its possible sets of non-conjugate Sylow subgroups? It is well known that a necessary and sufficient condition that a group is the direct product of its Sylow subgroups is that we arrive at identity by forming the successive groups of inner isomorphisms, but no general criterion as regards whether a group is a product of a set of non-conjugate Sylow subgroups seems to have been found.

\section{THE MATHEMATICS OF MAHĀVĪRĀCĀRYA.}

The Ganita-Sāra-Sangraha of Mahāvīrācārya with English

Translations and Notes. By M. RANंGĀCĀRYA, M.A., Rao Bahadur, Professor of Sanskrit and Comparative Philology in the Presidency College, and Curator of the Government Oriental Manuscripts Library, Madras. Sanskrit text and English translation. Madras, Government Press, 1912. $27+325 \mathrm{pp}$.

IT was announced at the Fourth International Congress of Mathematicians, at Rome, in 1908, that Professor Rangācārya had for a number of years been engaged in the laborious task of translating a work of great importance in the history of mathematics, the Ganita-Sāra-Sangraha of Mahāvīr the Learned. Now, after four years more, the work has been brought to completion, and the mathematical world is the debtor to Professor Rangācārya for his arduous labor and to the Government Press for publishing the volume that is before us.

We have so long been accustomed to think of Pataliputra on the Ganges and of Ujjain over towards the western coast of India as the ancient habitats of Hindu mathematics, that we experience a kind of surprise at thinking that other centers equally important existed among the multitude of cities of that great empire. We have known for a century, thanks chiefly to the labors of such scholars as Colebrooke and Taylor, 